\title{
DIGITAL WORKFLOW FOR THE CONSERVATION OF BAHRAIN BUILT HERITAGE: THE SHEIK ISA BIN ALI HOUSE
}

\author{
Luigi Barazzettia , Davide Mezzino ${ }^{\mathrm{b}, \mathrm{c}}$, Mario Santana Quintero ${ }^{\mathrm{b}}$
}

\author{
a ABC Department, Politecnico di Milano, Via Ponzio, 3120133 - Milan, Italy, luigi.barazzetti@polimi.it \\ b Carleton Immersive Media Studio (CIMS), 1125 Colonel by drive, Ottawa, On, K1S 5B6 Canada, mario.santana@carleton.ca, \\ davide.mezzino@gmail.com \\ c Interuniversity Department of Regional and Urban Studies and Planning (DIST), \\ Politecnico di Torino, via Sant’Ottavio, 28, 10122, Torino, Italy
}

KEY WORDS: Digital documentation, Conservation, Laser scanning, Photogrammetry, Processing workflow

\begin{abstract}
:
Currently, the commercial market offers several tools for digital documentation of historic sites and buildings. Photogrammetry and laser scanning play a fundamental role in the acquisition of metric information, which is then processed to generate reliable records particularly useful also in the built heritage conservation field. Although potentially very fast and accurate, such techniques require expert operators to produce reliable results, especially in the case of complex and large sites.

The aim of this paper is to present the digital workflow developed for data acquisition and processing of the Shaikh Isa Bin Ali house in Muharraq, Bahrain. This historic structure is an outstanding example of Bahrain architecture as well as tangible memory of the country history, with strong connotations in the Bahrain cultural identity. The building has been documented employing several digital techniques, including: aerial (drone) and terrestrial photogrammetry, rectifying photography, total station and laser scanning. The documentation project has been developed for the Bahrain Authority for Culture and Antiquities (BACA) by a multidisciplinary team of experts from Carleton Immersive Media Studio (CIMS, Carleton University, Canada) and Gicarus Lab (Politecnico di Milano, Italy).
\end{abstract}

\section{INTRODUCTION}

The documentation project presented in this work has been developed within the framework of the so-called 'Pearling initiative' financed by the Islamic Development Bank (IDB) to support the Conservation and Urban Economic Revival Project in the city of Muharraq, Bahrain (Economist Newspaper, n.d.). The Project involves the rehabilitation and conservation of twelve historical buildings, listed in the Pearling, Testimony of an Island Economy UNESCO site, inscribed in the World Heritage List in 2012 (UNESCO, n.d.).

Additionally to these buildings, a number of properties of significant historic value would also be conserved. Among these properties is the Shaikh Isa bin Ali House (Figure 1), an outstanding example of Gulf Islamic architecture (Bahrain Authority for Culture and Antiquities, n.d.).

The house dates back to the early XIX century and has been the residence of the Bahrain ruler from 1869 to 1932. After that it was passed down to the family and it was finally abandoned in 1973 because the dwellers moved to another city, Riffa (Bahrain Authority for Culture and Antiquities, n.d.).

Even if in the late '70s due to its advanced state of decay it was going to be demolished (1976) to build a public square, the director for museum and archeology, Shaikha Haya bint Ali AlKhalifa, at that time under the ministry of education, decided to take the property of the building to later conserve it (Bahrain Authority for Culture and Antiquities, n.d.).

The project included several conservation stages. Currently, the Bahrain Authority for Culture and Antiquities (BACA) is taking care of its conservation and maintenance.

The digital documentation of the house, required to develop informative conservation and maintenance actions, paid particular attention not only to shape, geometry and colour
(Mezzino, Quintero, Pwint, Latt, \& Rellensmann, 2016) but also the decorative apparatus, spatial design and materials employed as well as to the innovative building solution adopted to adapt to the local hot climate (i.e. ventilation strategies through wind tower, ventilation systems such as the badgir, etc.).

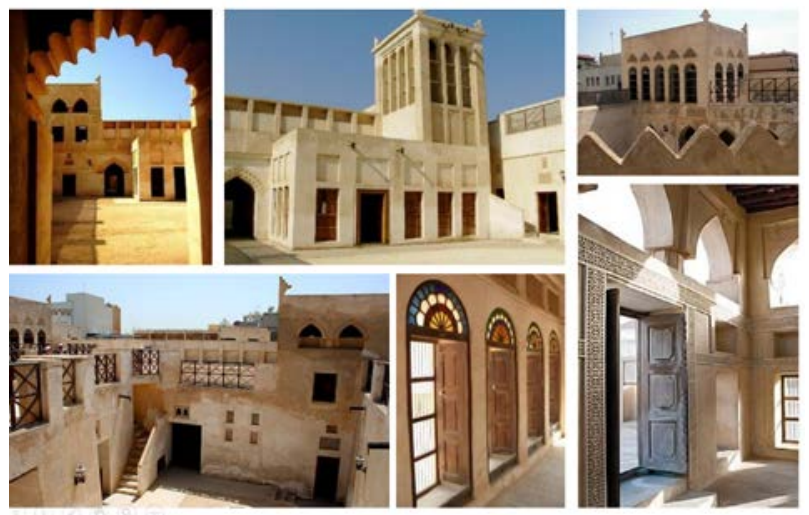

Figure 1 Photos illustrating the articulated architecture of the Shaikh Isa bin Ali House.

The scope of the digital documentation was to produce plans, sections, elevations (i.e. measured drawings) and digital orthophotos in a relatively short period of time. The available time for the completion of the project was about 6 weeks, including the time for the survey. This required an intense working week on site where images, scans and total station measurements were acquired and then processed in the office. The procedures, software, and tools used for image acquisition and processing are illustrated and discussed in the next section. 


\section{DATA ACQUISITION AND PROCESSING}

The limited time for the survey (5 days) required the combined use of different techniques for digital documentation. Photogrammetry and laser scanning were the techniques used to collect the geometric information for the production of the required deliverables (plans, cross sections, elevations, and orthophotos).

A flowchart of the used tools is shown in Figure 2, which illustrates a traditional approach for data acquisition and processing.
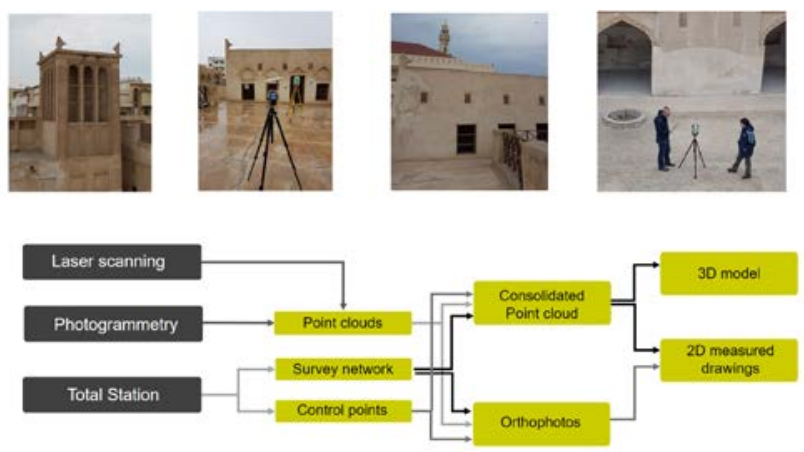

Docuremition tromates

DoCuREIATION OUTCOHES

Figure 2 Flowchart of the documentation workflow.

\subsection{Measurement and adjustment of a geodetic network}

The geodetic network was measured with a Leica 1203 total station. It consists of station points around the house and has multiple connections with rooms at different floors. The network (Figure 3) was not adjusted in a single step, but it is made up of a zero-order network measured the first day of the survey, which was then adjusted to determine the coordinates of points assumed as fixed for the following sub-networks. This "preliminary" network allowed one to establish a stable reference system right after the first day of the survey, so that its points were already considered as fixed for the progressive acquisition of laser scans. The zero-order network was adjusted with a free-network, i.e. using inner constraints to get the most precise solution. Starting from the zero-order network, different sub-networks were measured during the following days. They were adjusted fixing the point of the zero-order network to preserve the global reference system (Barazzetti, Banfi, Brumana, Raffaella Gusmeroli, \& Previtali, Mattia Schiantarelli, 2015).

The overall achieved precision was about $\pm 2-3 \mathrm{~mm}$, considering not only station points but also laser targets measured without multiple intersections.

The solution based on a zero-order network and other subnetwork progressively encapsulated into the first one allowed us to begin the registration of laser scans right after the end of the first working day.

This choice has been supported by the limited time for the survey and the impossibility to integrate the survey with a second measurement campaign. Acquiring the data during the day and processing them during the night was the solution chosen to guarantee a complete data acquisition in Bahrain, whereas the production of the deliverables were carried out in the offices (Ottawa - Canada and Milan - Italy) of the two universities involved.

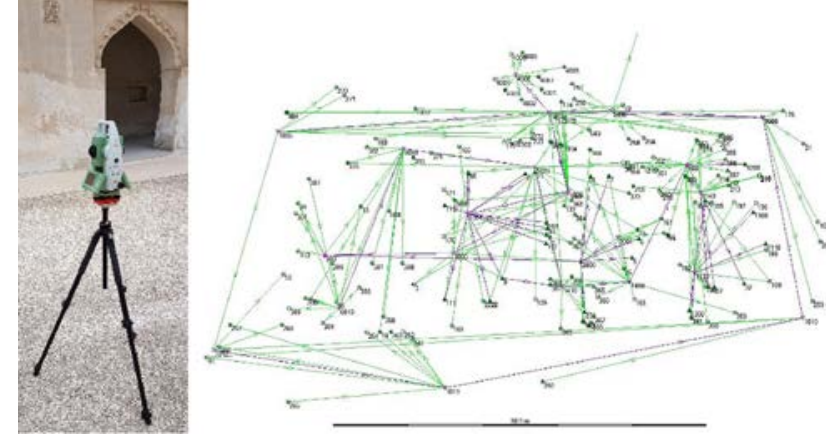

Figure 3 The scheme of the final geodetic network, which includes all the station points measured in different days.

\subsection{Laser scanning data acquisition and registration}

A set of 209 laser scans was acquired with a Faro Focus 3D. Data acquisition required 4.5 working days. Scans registration (Vosselman and Maas, 2010) was carried out using total station points as well as spherical targets. Additional checkerboard targets not measured with the total station were included as scan-to-scan correspondences. Scans were registered (Figure 4) with an overall precision of about $\pm 3-4 \mathrm{~mm}$, which is more than sufficient for the production of the required deliverables at a scale 1:50.

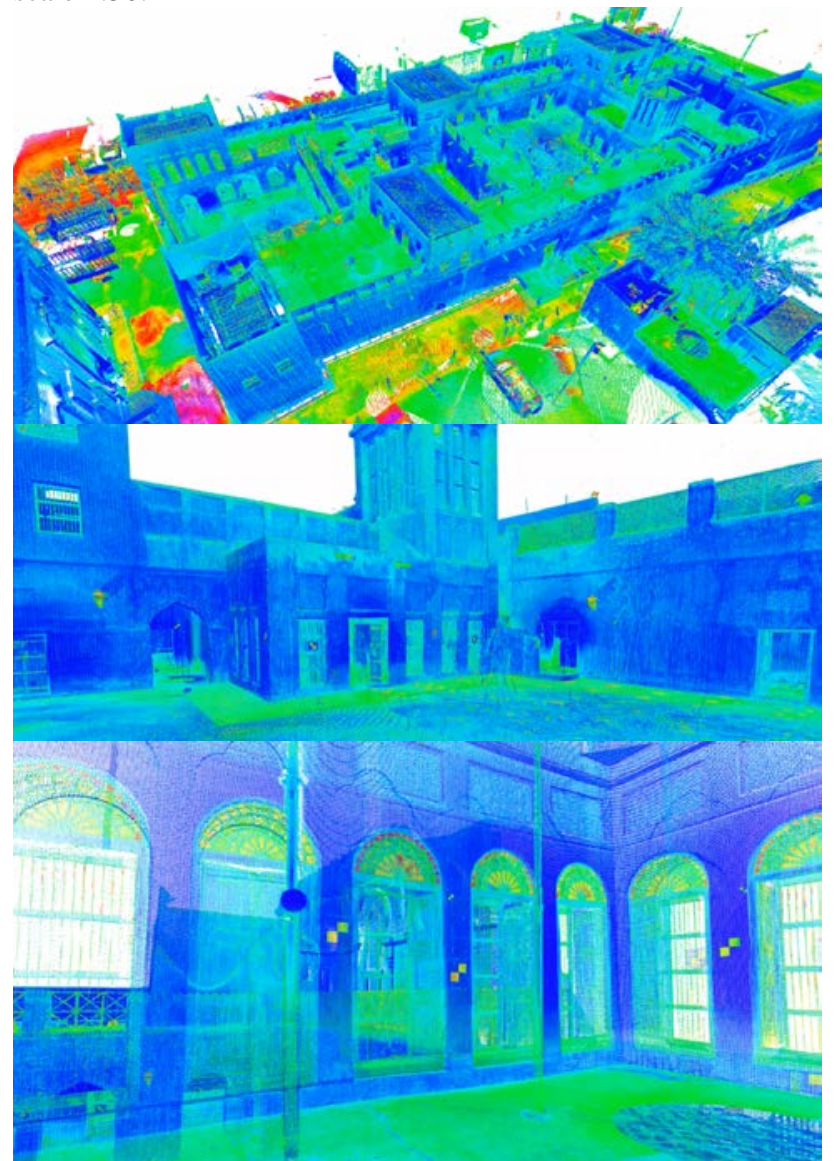

Figure 4 Images of the registered laser scans. Top: an overall view of the house, middle: the point cloud of the biggest courtyard, bottom: a detail of a room at the first floor.

As mentioned in the previous section, the registration was carried out right after the end of a single working day using the coordinates of the zero order geodetic network. Scans were 
mainly acquired with colour information, except when illumination conditions were not considered sufficiently good, such as in dark rooms or after the sunset.

\subsection{Digital photogrammetry}

Digital photogrammetry (Luhmann et al., 2006; Kraus, 2008) was used to generate orthophotos of external elevations, facades of internal courtyards, and rooms (including walls and ceilings). This required the acquisition of more than 5,000 images.

The used camera is a Nikon D610 equipped with $20 \mathrm{~mm}$ and 35 mm lenses. Both lenses were calibrated beforehand, so that calibration parameters were fixed during photogrammetric processing. The approach used for camera calibration is the target-less method proposed in (Barazzetti et al., 2011) and (Stamatopoulos and Fraser, 2014).

Rooms were processed in different ways depending on their geometry. In some cases the walls were considered as different photogrammetric projects, which can be combined by using points measured with total station and laser scanning. Some rooms were instead reconstructed with a single photogrammetric project that combines walls and ceiling in a single project. The choice between these two solutions depended on the avaibility of ground control points measured with the total station.

In the case of small and narrow rooms, a fisheye lens (16 mm) was used to reduce the number of images. Image acquisition was carried out with more images than those strictly necessary for the reconstruction. Indeed, the very large distortion close to the image edges did not allowed to produce orthophotos with a uniform resolution. More details about this effect can be found in Barazzetti et al., 2017.

An example of the second solution is illustrated in Figure 4. The required scale for such products was 1:20 but the final images have a much higher resolution, usually in the order of scale 1:5. The quality of the final orthophoto was not degraded to meet the basic requirement of a scale 1:20 (say a pixel size of about 2 $\mathrm{mm}$ ), preserving as much as possible the resolution of the images acquired.

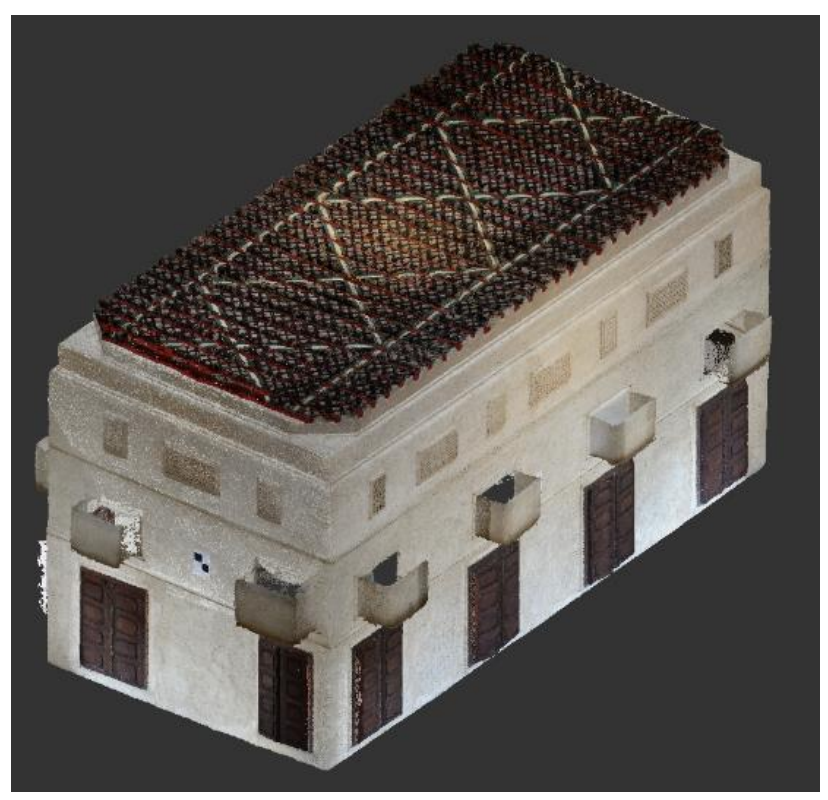

Figure 5 Point cloud of a room entirely generated from photogrammetry.
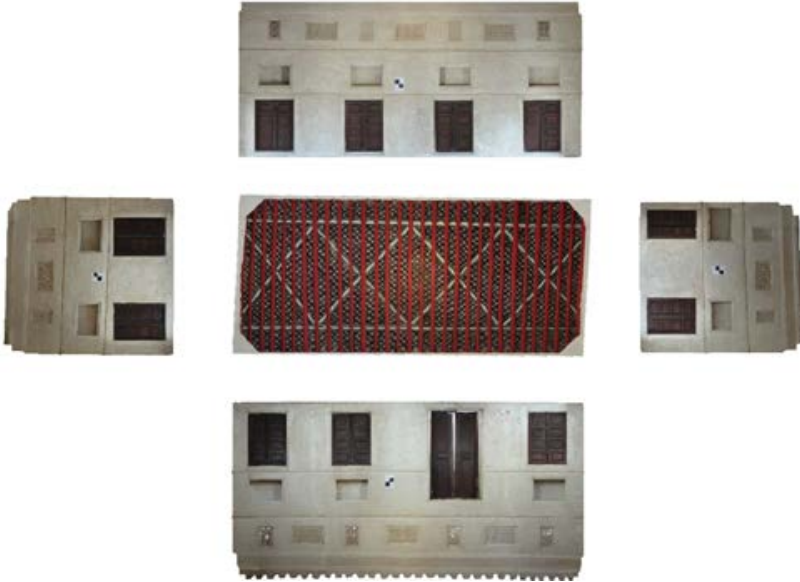

Figure 6 A room reconstructed with digital photogrammetry and the corresponding orthophotos of walls and ceiling.

Orthographic projections of elevations (Figure 6) were generated from sequences of images taken along a straight trajectory. Additional convergent images were acquired for irregular parts, such as the decorated doors. Ground control points were acquired from laser scanning point clouds to obtain a reference system consistent with the geodetic network.
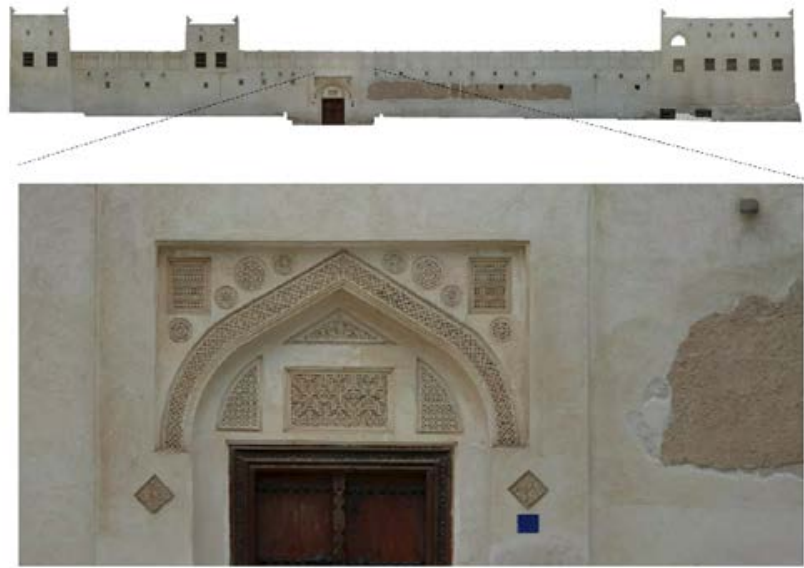

Figure 7 Example of orthographic elevation of the north façade.

Finally, a photogrammetric flight was conducted with a Phantom 3 Pro. A set of 198 images with a normal configuration was acquired along different strips. Images have a large overlap (about $85 \%$ ) and were acquired in about ten minutes. Images processing was carried out with ContextCapture, including some control points measured on the ground through a total station. The final metric accuracy on check points was better than $1 \mathrm{~cm}$. An example of the resulting point cloud from photogrammetric processing and the orthophoto is shown in Figure 8.

Overall, photogrammetric data processing was carried out with just two software:

(i) Agisoft PhotoScan, which was used for the images acquired inside the room;

(ii) Bentley ContextCapture, which was instead used for such as large blocks or very long image sequences. 

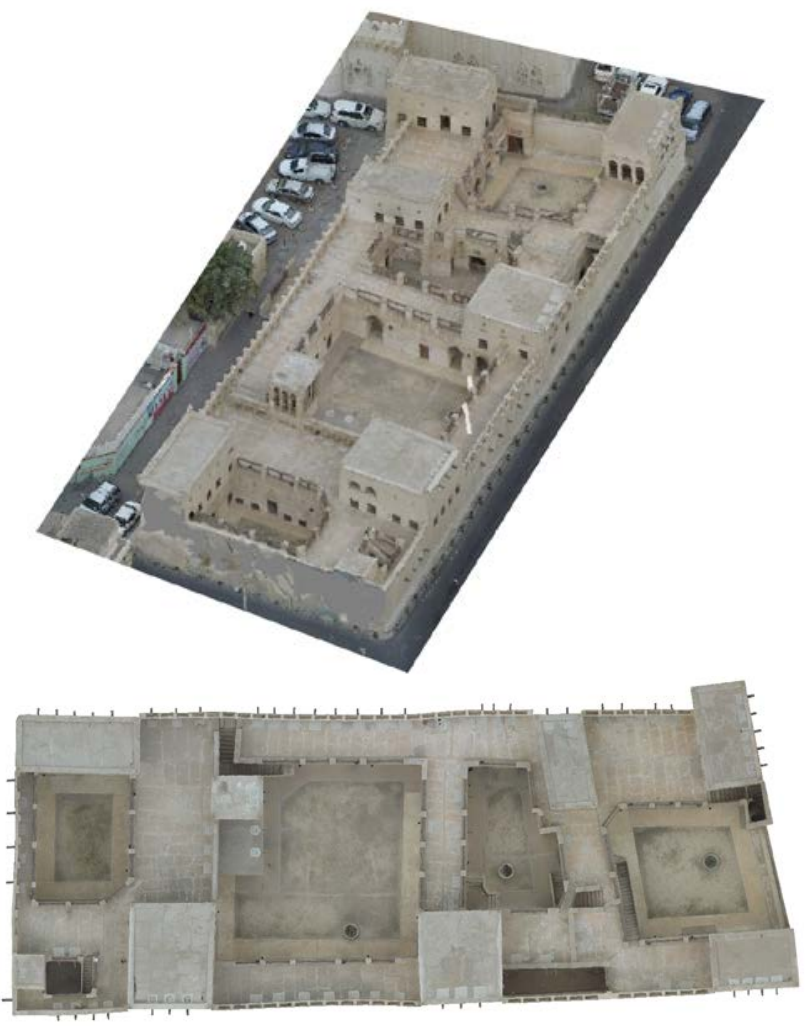

Figure 8 The point cloud extracted from aerial images (drone) and the final orthophoto.

\subsection{From point clouds to measured drawings}

Laser scanning and photogrammetric point clouds were used to create measured drawings at a scale 1:50 of the main house and the coffee house. This required the conversion of the different formats into a final point clouds in Autodesk Recap, so that the production of the different deliverables was carried out in AutoCAD (manual tracing using point clouds). The required deliverables were plans, sections and elevations for both the main house and coffee house. The creation of output project boards required intensive manual effort, being essentially based on the creation of point cloud slices with a limited thickness (usually $1 \mathrm{~cm}$ ) and manual tracing in AutoCAD. Examples of the generated deliverables are shown in Figure 9 and Figure 10. As (expert) operators, who did not participate to the survey, also produced the measured drawings, a $360^{\circ}$ videos was acquired walking through the different rooms of the building.

The camera used is a Samsung Gear 360, which is a low cost sensor equipped with two fisheye lens (front and rear facing images, i.e. a dual 15MP CMOS sensor with integrated f/2.0 fisheye lenses, dual cam video resolution of $2840 \times 1920$ pix, and dual cam photo resolution of $7776 \times 3888$ pix). The camera requires a Bluetooth connection with a Samsung mobile phone (such as the Samsung S6 or S7) to get real-time visualization and control acquisition parameters. Although the camera can be used as a standalone tool, the connection with the mobile phone is surely more user friendly and allows the user to check the quality of the acquired images, especially with bad illumination conditions.

The images can be downloaded from the camera as circular fisheye images or equirectangular projections for $360^{\circ}$ visualizations. A video of about 18 minutes was acquired walking through the different rooms of the building (Fig. 10), using Gear 360 ActionDirector to generate a panoramic video.

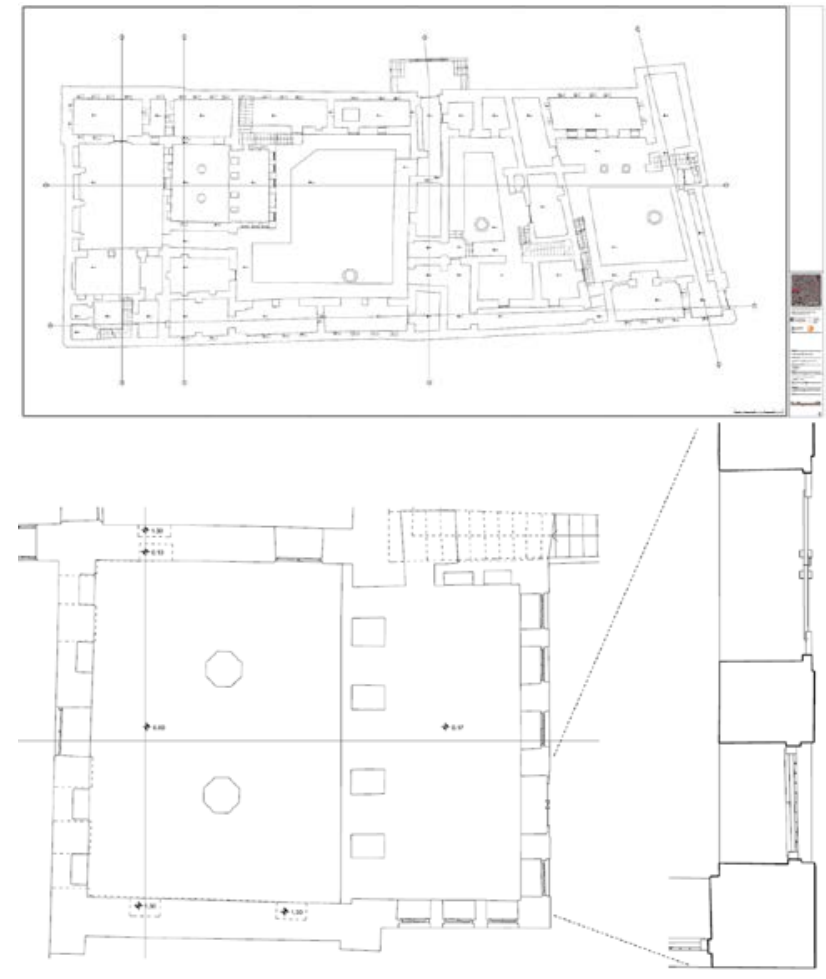

Figure 9 Examples of plan and detail that shows the level reached for the representation of small details, such as windows and doors.

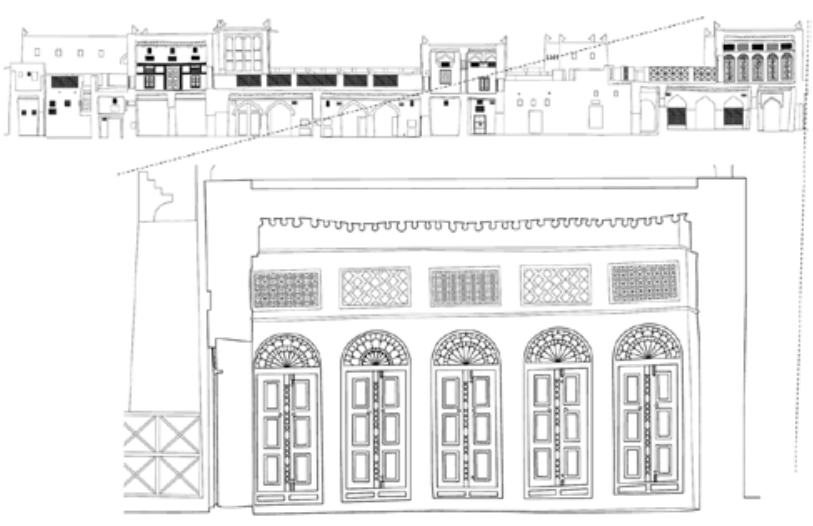

Figure 10 Example of cross section and local detail that shows the geometric irregularity of the building.

This allowed operators who did not visit the house to have a better understanding of the building and its condition. Such very simple solution demonstrated to be very effective in the interpretation of different constructive elements (especially small details), which were not visible in laser scanning point clouds, especially while tracing in AutoCAD. Considering the very limited cost of such sensors and the simple and user friendly acquisition and processing procedure, as well as the avaibility of different free software for $360^{\circ}$ video visualization (the software used is GoPro VR player), such additional product will be acquired also in future work. 


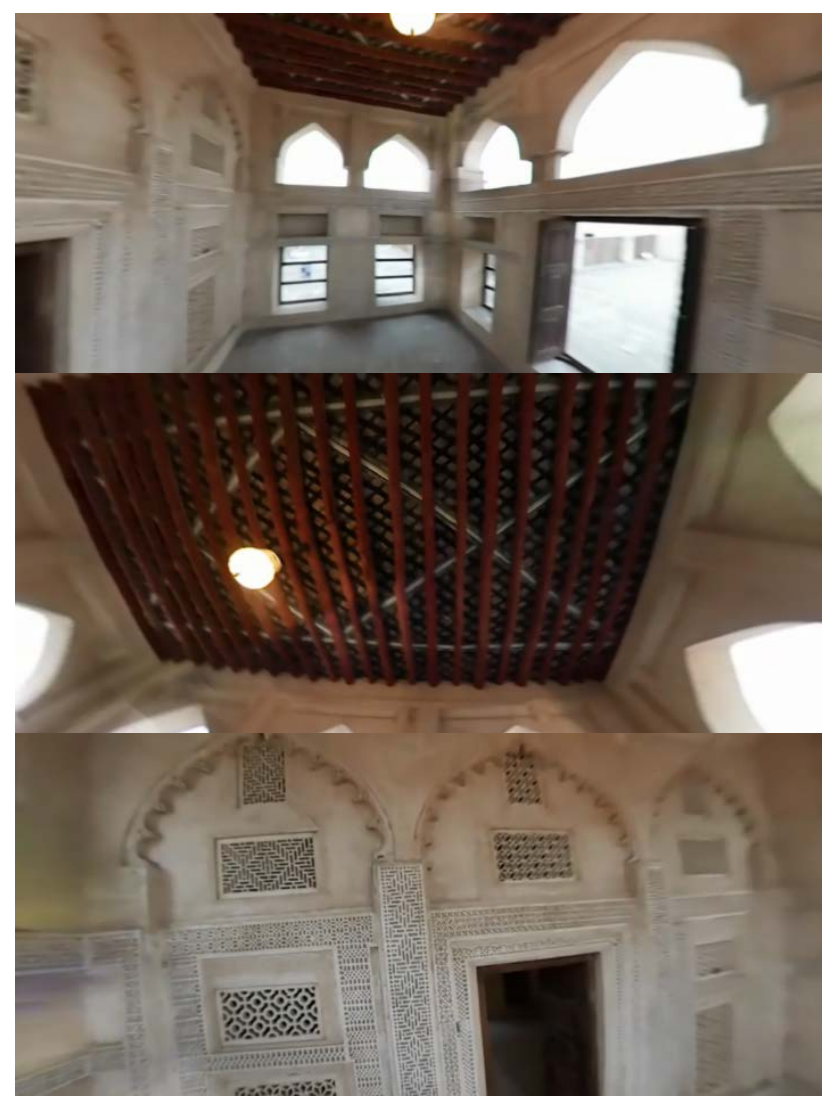

Figure 11 Some frames of the panoramic video acquired inside a room. Although the resolution is quite limited, it provide an immersive visualization to understand the building while tracing in AutoCAD.

\section{CONCLUSIONS}

The paper presented the digital documentation work of the Isa Bin Ali house, in Bahrain. The proposed workflow is based on different tools and methods for data acquisition and processing, mainly based on photogrammetry and laser scanning.

A geodetic network adjusted via least squares provided a set of control points, i.e. a reference system for both laser scanning and photogrammetric techniques. The use of digital photogrammetry was mandatory to generate accurate digital orthophoto, for which the resolution and quality of those achievable via laser scanning (orthophoto from colored point cloud) is not sufficient for accurate products. The production of the measured drawings was carried out with laser scanning point clouds, which were then integrated with photogrammetric point clouds and digital orthophotos (mainly for the elevations). Integration of photogrammetry and laser scanning was necessary to overcome traditional problems during scan acquisition, such as occluded areas and reconstruction of small details.

Overall, the projected was conducted with 9 software to solve for the following tasks:

- Leica Geo Office: adjustment of total station measurements;

- $\quad$ Faro Scene: registration of laser scans;

- Agisoft PhotoScan: processing of images acquired inside the room;

- $\quad$ Bentley ContextCapture: processing of large images blocks (e.g., images from drone);
- Autodesk Recap: generation of point clouds in the AutoCAD format and basic point cloud editing;

- Autodesk AutoCAD: tracing of measured drawings from point clouds;

- $\quad$ Bentley Pointools: point cloud editing and point cloud visualization;

- $\quad$ Gear 360 ActionDirector: creation of the $360^{\circ}$ video acquired with the Samsung Gear 360;

- GoPro VR player: visualization of $360^{\circ}$ videos.

Although the proposed workflow for digital documentation is not novel, we would like to stress the attention on the aspects related to the integration of different measurements, from different sensors, processed in different software. The opportunity to use a single reference system given by total station data (instrument sometimes neglected in documentation projects) remains a rapid, efficient, reliable and accurate solution to combine all the data necessary for the production of the measured drawings.

\section{ACKNOWLEDGEMENTS}

The author wants to thank the Bahrain Authority for Culture and Antiquities (BACA), which sponsored the digital documentation project. We want also to thank all the people who participated to survey, data processing and production of measured drawings: Fabio Roncoroni, Mattia Previtali, Daniela Oreni, Branka Cuca, Sara Caspani, Chiara Midali, Lori Chan.

\section{REFERENCES}

Barazzetti, L., Mussio L., Remondino F., Scaioni M., 2011. Targetless camera calibration. Int. Archives of Photogrammetry, Remote Sensing and Spatial Information Sciences (IAPRSSIS), 38-5/W16, March 2-5, Trento, Italy

Barazzetti, L., Previtali, M., Roncoroni, F., 2017. Fisheye lenses for 3d modeling: evaluations and considerations. Int. Int. Arch. Photogramm. Remote Sens. Spatial Inf. Sci., XLII-2/W3, 79-84, 2017

Bahrain Authority for Culture and Antiquities. (n.d.). Sheikh Isa Bin Ali House. Retrieved from http://culture.gov.bh/en/visitingbahrain/destinations/Name,1055 4,en.html\#.WSRNnWjytPY

Barazzetti, L., Banfi, F., Brumana, Raffaella Gusmeroli, G., \& Previtali, Mattia Schiantarelli, G. (2015). Cloud-to-BIM-toFEM: Structural simulation with accurate historic BIM from laser scans. Simulation Modelling Practice and Theory, 57, 7187.

Economist Newspaper. (n.d.). Muharraq Pearling Heritage Conservation and Urban Economic Revival. Retrieved March 21, 2017, from http://jobs.economist.com/job/10786/generalprocurement-notice/

Kraus, K., 2008. Photogrammetry: Geometry from Images and Laser Scans. Second edition.Walter de Gruyter. 459 pages.

Luhmann, T., Robson, S., Kyle, S. and Harley, I., 2006. Close Range Photogrammetry: Principles, Techniques And Applications. John Wiley \& Sons. 510 pages. 
Mezzino, D., Quintero, M. S., Pwint, P. M., Latt, W. T. H., \& Rellensmann, C. (2016). Technical assistance for the conservation of built heritage at bagan, Myanmar. International Archives of the Photogrammetry, Remote Sensing and Spatial Information Sciences - ISPRS Archives, 41(July), 945-952. https://doi.org/10.5194/isprsarchives-XLI-B5-945-2016

Stamatopoulos, C., Fraser, C.S., 2014. Automated Target-Free Network Orientation and Camera Calibration. Int. Arch. Photogramm. Remote Sens. Spat. Inf. Sci. 2014;1:339-346

UNESCO. (n.d.). Pearling, Testimony of an Island Economy. Retrieved March 2, 2017, from Pearling, Testimony of an Island Economy

Vosselman, G. and Maas, H.G., 2010. Airborne and Terrestrial Laser Scanning. Whittles Publishing, Caithness, Scotland (UK).

Agisoft PhotoScan: http://www.agisoft.com

Bentley ContextCapture: https://www.acute3d.com/

Autodesk AutoCAD and Recap: https://www.autodesk.com/

Leica Geo Office: leica-geosystems.com

Faro Scene: www.faro.com

Bentley Pointools: https://www.bentley.com

Gear 360 ActionDirector: www.samsung.com

GoPro VR player: www.kolor.com/gopro-vr-player/download/ 